\title{
Perfusion Index: Physical Principles, Physiological Meanings and Clinical Implications in Anaesthesia and Critical Care
}

Short title: Clinical Usefulness of Perfusion Index

Maxime COUTROT MD ${ }^{1,4}$, Emmanuel DUDOIGNON MD ${ }^{1,2 *}$, Jona JOACHIM MD ${ }^{1}$, Etienne GAYAT MD, PhD 1, 2, 3, 4, Fabrice VALLEE MD, PhD 1, 2, 3, 4, 6, 7, François DEPRET MD, PhD 1, 2, 3, 4, 5

(1) AP-HP, GH St-Louis-Lariboisière, Department of Anaesthesiology and Critical Care and Burn Unit, Paris, France

(2) University Paris Diderot, France

(3) UMR INSERM 942, Institut National de la Santé et de la Recherche Médicale (INSERM)

(4) FHU PROMICE, Paris, France

(5) F-CRIN INICRCT network- Paris, France

(6) Inria, France

(7) LMS, Ecole Polytechnique, CNRS, Institut Polytechnique de Paris, France

*Corresponding author: Emmanuel DUDOIGNON

Department of Anaesthesiology and Critical Care and Burn Unit, Saint-Louis Hospital, 1, Avenue Claude Vellefaux, Paris, France

Email : emmanuel.dudoignon@aphp.fr

Keywords: Perfusion index; Haemodynamic monitoring; non-invasive monitoring; photoplethysmography

Disclosure of funding: None 


\section{Conflicts of interest:}

A patent application ( $\mathrm{n}^{\circ}$ PCT IB2009/006903) is pending on variations of $\mathrm{PcCO}_{2}$ and PI during Heating Challenge. The patent belongs to the Assistance Publique-Hôpitaux de Paris (France).

Etienne Gayat has received Consulting Fees from Magnisense, research support from Sphingotec, Deltex Medical and Retia Medical.

Fabrice Vallée has received research support from Radiometer, Deltex Medical and Retia Medical.

François Dépret has received research grants from the French Ministry of Health, SFAR (Société française d'anesthésie et de reanimation), the ESICM (European Society of Intensive Care Medicine), and lecture fees Sedana medical.

\section{Authors' contribution:}

Maxime Coutrot: this author drafted and approved the final version of the manuscript Emmanuel Dudoignon: this author drafted and approved the final version of the manuscript Jona Joachim: this author drafted and approved the final version of the manuscript Etienne Gayat: this author drafted and approved the final version of the manuscript Fabrice Vallée: this author drafted and approved the final version of the manuscript François Dépret: this author drafted and approved the final version of the manuscript All the authors have drafted and approved the final version of the manuscript.

\section{Glossary of Terms:}

PPG: photoplethysmography

PI: perfusion index

PVI: pleth variability index

AC: alternating current

DC: direct current

SV: stroke volume

MAP: mean arterial pressure 
ICU: intensive care unit

ScvO2: central venous oxygen saturation

$\mathrm{P}(\mathrm{v}-\mathrm{a}) \mathrm{CO}_{2}$ : arteriovenous carbon dioxide gradient

PLR: passive leg raising

$\mathrm{Cl}$ : confidence interval

\section{Acknowledgement}

The authors would sincerely thank $\mathrm{Dr}$ Colleen Breslin and Isabelle Taufflieb for their proofreading as well as their advice for writing this review 


\section{Abstract}

Photoplethysmography (PPG) has been extensively used for pulse oximetry monitoring in anaesthesia, perioperative and intensive care. However, some components of PPG signal have been employed for other purposes, such as non-invasive haemodynamic monitoring. Perfusion index (PI) is derived from PPG signal and represents the ratio of pulsatile on nonpulsatile light absorbance or reflectance of the PPG signal. PI determinants are complex and interlinked, involving and reflecting the interaction between peripheral and central haemodynamic characteristics, such as vascular tone and stroke volume. Recently, several studies have shed light on the interesting performances of this variable, especially assessing regional or neuraxial block success, and haemodynamic monitoring in anaesthesia, perioperative and intensive care. Nevertheless, no review has yet been published concerning the interest of $\mathrm{PI}$ in these fields.

In this narrative review will be exposed first the physiological and pathophysiological determinants of $\mathrm{PI}$, and then the mean to measure this value as well as its potential limitations. In the second part, the existing data concerning usefulness of PI in different clinical settings such as operating theatres, intensive care units and emergency departments will be presented and discussed. Finally, the perspectives concerning the use of PI and mentioned aspects that should be explored regarding this tool will be underlined. 


\section{1) Introduction}

In the 1970s, following previous works, Takuo Aoyagi, who passed away on the $18^{\text {th }}$ of April 2020, developed pulse oximetry using red and infrared lights transmission through tissues [1]. Increasing interest in non-invasive monitoring of macrohaemodynamic and microcirculation has risen in anaesthesia, perioperative and critical care. Photoplethysmography (PPG) signal, which was used during decades only to monitor oxygen saturation, represents nowadays an interesting tool in haemodynamic monitoring.

PPG is a non-invasive tool, cheap, fast, and simple to use. However, interpretation of PPG data can be challenging: haemodynamic monitoring using PPG signal initially suffered from a large lack of knowledge and misunderstanding of its determinants, resulting in an incorrect use of this multifactorial device. However, analysis of PPG signal has benefitted from regained interest in the past years and the usefulness of PPG has been highlighted by several works in anaesthesia, perioperative and critical care [2-4].

Among different components of PPG signal, perfusion index (PI), which represents the proportion of the pulsatile part of PPG, has been widely studied and stands as a promising tool for physicians. A good understanding of the principles of PPG and PI determinants as well as the knowledge of its limits is therefore essential to optimise its correct use and performance. Physical principles and the physiological determinants of PI will be explained thereafter in this review.

Several reviews have been proposed on PPG, notably about PI respiratory variations, commonly referred to as Pleth Variability Index (PVI), and its applications [3,5-7]. However, 
none of above-mentioned reviews focused on PI itself. Additionally, several new data have been published since.

This review covers the physiological and technical aspects of PPG and PI that clinicians need to understand and presents the clinical usefulness of $\mathrm{PI}$ in the operating theatre, perioperative and critical care.

\section{2) How is Perfusion Index measured?}

\subsection{Principle of photoplethysmography}

The principle of conventional PPG is based on indirect measurement of tissue volume variations by absorbance variations of light beams through this tissue. The oximeter probe generates incident red and ultra-red light beams, whose transmitted intensities are transformed into an electrical current by a photodetector, after penetrating a tissue (Figure 1) $[8,9]$.

Another PPG modality (PPG by reflection) uses reflection properties of the light in the tissue, according to the same principle. The photodetector is then placed next to the light source to measure the reflectance of the incident beams [10].

Usually, two wavelengths are used for PPG: red light (660 nm) and infrared light (940 nm), mainly absorbed by deoxyhaemoglobin and oxyhaemoglobin, respectively. The PPG curve is obtained with infrared light absorption variations. However some PPG devices use one, or more often more than two wavelengths [11]. 


\subsection{PI components: alternating and direct currents}

Light absorption varies across the cardiac cycle. The absorption is maximal during the systole, reflecting the dilatation of vessels under the systolic pressure, i.e., the increase of arterial blood volume under the light source. The signal received by the photodetector is then decomposed into pulsatile and non-pulsatile signals. Pulsatile variations in light absorption, during systole, are commonly referred to as "alternating current" (AC). AC represents variations of absorbance or reflectance of the incident light beams due to pulsatile vessels under arterial pressure variations, i.e. the sum of the variations of the diameters of pulsatile vessels through which the light beams pass. AC then represents a volume variation measurement. It is important to underline that $A C$ is therefore not a flow measurement in those vessels but an indirect measurement of the arterial volume variation during the cardiac cycle.

The PPG curve displayed on actual monitors represents AC, derived from the infrared light signal, after signal processing. Restitution of AC requires computer processing of the raw signal received by the photodetector by computer filters to reduce signal artifacts $[8,12,13]$. These manufacturer-dependent algorithms can significantly deform the PPG curve from one manufacturer to another $[8,9]$.

On the other hand, continuous absorption is referred to as "direct current" (DC), from which AC varies. DC corresponds to light absorption from other tissues, such as non-pulsatile capillaries and venous vessels, skin, soft tissues, and bones. DC is not displayed in current practice on usual oximeter's monitors. 


\subsection{PI calculation: alternating and direct currents ratio}

PI represents the ratio of pulsatile light absorption on continuous light absorption, i.e., the ratio $A C / D C$ (Figure 1). PI, often referred to as "peripheral PI" was initially used as a quality signal indicator for pulse oximetry $[4,14]$. However, PI represents the local blood volume variation during systole, and varies according to the systemic and local haemodynamic status. Hence, PI can be used for non-invasive haemodynamic monitoring.

\subsection{Physiological value of PI}

In healthy awake volunteers, when measured at the finger, the mean PI values ( \pm standard deviation) described in two studies were $2.2 \% \pm 2.0$ and $3.5 \% \pm 2.4[15,16]$. This means that $A C$ represents only around $2 \%$ to $3 \%$ of $D C$, and by analogy in vascular physiology, it means that the blood volume under the sensor increases by around $2 \%$ at each heartbeat. However, due to the wide variations in normal values in healthy volunteer (from $<1 \%$ to $>$ $10 \%)$, it is difficult to propose a reliable normal value of this parameter.

Determinants of $\mathrm{PI}$ are numerous and complex. When measured in a peripheral site, both $\mathrm{AC}$ and $\mathrm{DC}$ and their ratio, i.e., $\mathrm{PI}$, are the resultants of systemic and local factors, discussed below, and resumed in Table 1. The characteristics and main results of discussed studies are detailed in Supplementary Table 1.

\subsection{Influence of direct current (DC) determinants}

As $\mathrm{PI}$ represents the ratio AC/DC, any variation of DC will result in PI variation. Hence, soft tissues or venous compression (e.g., by a finger clip) may decrease DC and increase PI. Likewise, congestion due to global fluid overload would have the opposite consequences. Limb position due to changes of venous filling and thus DC would also affect PI value, with an 
increase in DC if the limb is in a declive position (venous congestion) and the opposite in a proclive position [3]. Physiologically, DC is also not constant, and small variations are observed due to variation of venous return and sympathetic tone in patients spontaneously breathing or under mechanical ventilation $[17,18]$. Similarly, DC may also change in case of change in vascular tone, as under action of vasoactive drugs (i.e., decrease of DC under increased vascular tone) [19].

\subsection{Alternating current $(\mathrm{AC})$ : vascular tone versus stroke volume}

Some authors have described PI as a surrogate of vascular tone only. Lima et al. showed that PI was correlated with central to toe temperature gradient - an accurate surrogate of vascular tone [15]. Indeed, PI rapidly increased after local vasodilation induced by plexus or epidural anaesthesia, measured in the blocked area. PI was also strongly influenced by changes of vascular tone under action of vasopressors in patients under general anaesthesia (i.e., decrease in PI secondary to norepinephrine infusion) [20-25].

Indeed, several recent studies suggested that stroke volume (SV) is another important determinant of PI. PI was correlated to superior vena cava flow and cardiac output in infants [26-28]. Low flow could be associated with increased vascular tone, but such data are not presented in these works. Van Genderen et al. exposed healthy volunteers to a lower body negative pressure [29]. They observed a rapid decrease of PI (from 2.2\% [1.6-3.3] to 1.3\% [0.9-1.7], expressed as median and interquartile), while SV decreased, and skin temperature difference between forearm and fingertip did not increase significantly. Lack of significant variation in skin temperature gradient between forearm and fingertip may be explained by the lack of power of this study (the study population comprised 25 males) or because of temperature change inertia. This suggested that PI was not only influenced by local vascular 
tone but also by SV itself. Another study performed in healthy volunteers showed PI variations induced by body positioning modifications (e.g., Trendelenburg, 45-degree, supine etc.) [30]. The highest values of PI were observed in Trendelenburg position (7.8 $\pm 3.8 \%)$. On the contrary, $\mathrm{PI}$ was the lowest in the sitting position $(4.5 \pm 2.5 \%)$, suggesting a positive relationship between $\mathrm{PI}$ and SV. This has been recently confirmed in a work showing that variation of PI was highly correlated to variation of SV $(r=0.9)$, and mean arterial pressure (MAP) ( $r=0.9)$ during head up and down tilt test in patients under anaesthesia with low basal sympathetic tone or reactivity to position-induced hypovolaemia [31]. Additionally, other recent works further discussed, found significant correlation of cardiac index and PI variations after passive leg raising and fluid challenge [32-34]. These works confirmed the major influence of SV on PI, and the complexity of PI variations interpretation, especially in septic shock [35].

The respective influence of vascular tone and SV variations in case of vasoplegia are drawn in Figure 2, cases $\mathbf{A}$ and B. Figure 2 case $\mathbf{A}$ represents moderate vasoplegia (for example intraoperative hypotension secondary to anaesthetic drugs). In this case, the increase of PI (from 5 to 10\%) would mainly be the consequence of a fall of arterial vascular tone (secondary to vasodilatation induced by anaesthetic drugs) without substantial SV variations $[15,19,25]$. In case of severe vasoplegia (Figure $\mathbf{2}$ case B), such as under general anaesthesia with overdose of anaesthetics leading to a significant decrease in stressed volume, venous return and thus in SV, or in case of surgical bleeding, PI decreases secondarily in comparison to Figure $\mathbf{2}$ case A despite a low arterial vascular tone. In this case, SV would be the predominant determinant, leading to PI secondary decrease, i.e. "hiding" the effect of the decrease of vascular tone, on PI [29-31,33]. However, data are lacking to quantify the 
respective contributions of vascular tone and stroke volume in $\mathrm{PI}$ values and interpret its variations in such clinical situations.

On the contrary, as illustrated in the Figure $\mathbf{2}$ case $\mathbf{C}$, in case of hypovolaemia with preserved baroreceptors activation, SV will decrease and vascular tone increase. Both variations of SV and vascular tone will contribute to a decrease of AC. In such conditions, a decrease of DC secondary to the activation of the sympathetic system also participates to the drop of $\mathrm{PI}$, suggesting that the decrease in $A C$ is predominant over the decrease in $D C[19,29,30]$. Another comparable situation characterised by a low SV and potential increased vascular tone is cardiac failure, where both contribute also to the fall of AC, DC, and PI values (Figure 2 case D). In this situation, PI would be typically extremely low PI $(<0.5)$. After inotropic drugs, an increase in SV would lead to a greater PI value [19].

\subsection{Local conditions}

PI measurement assesses local perfusion. The PI value varies according to the measurement site, even for clothe sites to a few centimetres [33]. Thus, PI is highly influenced by systemic macrohaemodynamic status as well as local conditions. Since local vascular tone is mainly influenced by thermoregulation and non-thermoregulatory stimuli (e.g, nociception, exercise...) [36,37]. As an example, PPG waveform variation was substantially different between ear and finger after cold exposure [38]. Regional anaesthesia also illustrates the influence of local conditions: the increase in PI measured in the blocked area after regional anaesthesia is the witness of a decrease in local vascular tone, associated with an increase in local blood flow and volume at each heartbeat in the blocked region. Both the decrease in vascular tone and the increase in local blood flow and volume are contributing to the rise of $A C$ and PI values. As for other variations of vascular tone, it is expected that DC also increase 
in the blocked area, although this has not been studied. In this situation, PI is a tool for local haemodynamic monitoring, but not central haemodynamic. Likewise, many local conditions must be considered to interpret PI value: local compression (soft tissues, veins and/or arteries), outside temperature variations, positions of the limbs or severe arterial abnormalities such as obliterating arteriopathy. In the conditions mentioned above, peripheral PI rather reflects local conditions than the central haemodynamic status $[11,27]$. However, no difference was found in basal PI in volunteers with or without vascular disease (hypertension and diabetes mellitus). On the contrary, macrohaemodynamic status (i.e., cardiac index) can influence PI value depending on the measurement site. Indeed, in infants, PI tends to be higher at the right hand compared to foot for low cardiac output, and inversely for high cardiac output [26]. All these elements suggest the importance of regional perfusion variation secondary to changes of sympathetic response and central haemodynamic status on local PI value.

\subsection{Sources of errors and variations of PPG signal}

One should also be aware of the classic and frequent sources of errors in the measurement of PI. Any extrinsic soft tissues and/or vascular compression could affect AC and/or DC and so the basal value of $\mathrm{PI}$ and/or its variations [3]. A common example is the digital compression by the clamp of PPG. The ear and finger PPG sensors are usually fitted with a clip, which prevents venous stasis and its influence on DC and thus on PI. Without a clip, forehead PPG waveform, i.e., AC signal, has been described significantly affected by a strong venous signal. Therefore, venous compression by a clip may result in a more reliable arterial waveform [39]. However, the use of an external pressure on the forehead probe with a dedicated headband to suppress the impact of venous pulsation may also result in a more 
reliable PPG waveform. Other classical sources of errors or factors that may influence PPG waveform, such as nail polish, obesity, age, gender, ambient lights changes, course artifacts of movement of the probe, or the patient, etc., have been recently pointed out [11].

\section{3) Clinical usefulness in Anaesthesia: what are the data?}

PI has been studied in the operating theatre for patients under regional or general anaesthesia in different clinical settings. The characteristics and main results of discussed studies are detailed in Supplementary Table 1.

\subsection{PI in assessment of regional block success}

At first, PI was used as an early indicator of regional anaesthesia success, using PI increase to detect vasodilation due to sympathetic tone inhibition.

PI measured at the toe was described as the earliest and $100 \%$ sensitive indicator of sympathectomy and epidural anaesthesia success compared to MAP and skin temperature $[21,40]$. Usefulness of PI variation after neuraxial anaesthesia in paediatric patients under general anaesthesia was also demonstrated [23,41]. PI variation was highly predictive of block success for regional plexus blocks in adults as well $[20,22,42]$. Variation of PI by a factor 1.55 had an area under the receiver operating characteristic curve of 0.94 for sciatic block, and area under the receiver operating characteristic curve of 1 for axillary block [20]. PI accuracy for prediction of regional block success was also higher than cold or pinprick sensations.

A recent work showed that PI performances for detection of regional block success were not different when using epinephrine as an adjuvant to local anaesthetics [43]. 


\subsection{PI in assessment of neuraxial anaesthesia-induced haemodynamic variations}

In parturients undergoing caesarean delivery after spinal-epidural anaesthesia, basal PI measured at finger location was correlated with the decline of systolic arterial pressure $(r=$ 0.66) [44]. Baseline PI also showed a better correlation with systolic arterial pressure or MAP decrease than baseline heart rate, systolic arterial pressure, or MAP. The area under the receiver operating characteristics curve for baseline PI to predict arterial hypotension after spinal-epidural anaesthesia (defined as decrease in systolic arterial pressure $\geq 25 \%$ ) was 0.87 $(95 \% \mathrm{Cl} 0.74-0.99, p<0.001)$, with a best threshold value of $3.5 \%$. Similar results were found later with the same cut-off value [45]. So, a PI value greater than $3.5 \%$ before spinal anaesthesia is a risk factor for anaesthesia-induced hypotension. One could expect that baseline PI value would also predict accurately diastolic arterial pressure decrease since vascular tone is one of the main determinants of diastolic arterial pressure. However, none of these studies evaluated the correlation between baseline PI values and diastolic arterial pressure decrease. A high PI value at baseline suggests a low basal vascular tone. Therefore, the decrease in sympathetic tone induced by spinal-epidural in patients with already low basal sympathetic tone, is more likely to induce a decrease in the stressed volume, resulting in a decrease of venous return and SV. Thus, PI is a simple tool to easily detect parturient patients with low sympathetic tone at high risk of arterial hypotension after spinal anaesthesia.

\subsection{PI in assessment of nociception}

As sympathetic tone is highly affected by nociceptive stimuli, PI signal has been used for nociception assessment during anaesthesia and in critical care [5]. Thus, the increase of vascular tone and MAP following nociceptive stimuli is associated with a decrease in PI [46]. 
Variation in PI could nevertheless be viewed only as a measurement of haemodynamic variation, i.e., increase of sympathetic tone, following nociceptive stimulation. Hence, it may not be accurate in patients with external control of adrenergic receptors, such as those under high dose of vasopressors or contrarily, in case of pharmacological blockade of their sympathetic tone (e.g., epidural anaesthesia or peripheral nerve blockade).

\subsection{PI to detect blood pressure variation during general anaesthesia}

Recent data have highlighted usefulness of PI to detect arterial hypotension during general anaesthesia. During induction of anaesthesia, PI variations were inversely correlated with MAP variations [25]. An increase of $51 \%$ or more from baseline PI detected a decrease of MAP of at least $20 \%$. Another recent study found opposite but complementary results regarding the association between MAP and $\mathrm{PI}$, in patients under general anaesthesia. Indeed, in 20 patients under general anaesthesia with head-up and head-down tilt, $\mathrm{H} \varnothing \mathrm{jlund}$ et al. observed that PI variations were positively correlated to MAP variations [31]. In this study, head-up tilt induced a decrease in SV correlated to MAP decrease, suggesting no significant variations in vascular tone, and resulting in a drop of PI. On the contrary, induction of anaesthesia is associated with severe drop in vascular tone. Both studies explored two major determinants of PI, reinforcing the fact that PI should be considered as the balance between SV and vascular tone, as schematically described in Figure 2. Then, a rapid variation of $\mathrm{PI}$ represents an alarm signal that should warn and force the clinician to quickly check the occurrence of an arterial hypotension in patients under general anaesthesia, monitored with intermittent cuff blood pressure measurement. Kinetics of PI variation may also help clinicians to identify the mechanism of arterial hypotension and manage these situations quickly and accurately: an increase of PI suggests excessive 
vasodilatation (relative to excessive depth of anaesthesia for example), while a secondary decrease of PI in a patient deeply anaesthetised may reveal SV decrease (hypovolaemia and/or cardiac dysfunction).

\subsection{PI and prognosis in anaesthesia}

In a large recent and retrospective study, intraoperative $\mathrm{PI}$ was associated with severe postoperative complications or death (lower values being associated with worse outcomes) in a time-dependent manner even after adjustment for confounding variables [47]. Furthermore, they observed that the association between PI with the primary outcome was higher in patients with MAP > $65 \mathrm{mmHg}$ compared to patients with MAP $\leq 65 \mathrm{mmHg}(\mathrm{OR}=$ 1.17; $95 \% \mathrm{Cl} 1.09-1.27 ; p<0.001$ vs. $\mathrm{OR}=1.07 ; 95 \% \mathrm{Cl} 1.02-1.13 ; p=0.011 ;$ respectively), suggesting a degree of incoherence between systemic haemodynamic and microcirculation. Indeed, increasing afterload with the use of vasopressors without assessing the cause of hypotension (e.g., hypovolaemia, cardiac dysfunction...) could impair tissue perfusion and therefore lead to organ dysfunction. Whether targeting PI during anaesthesia could improve patient's outcome remains to be assessed in randomised controlled trials.

\section{4) Clinical usefulness in ICU and emergency department: what are the data?}

The characteristics and main results of discussed studies are detailed in Supplementary

\section{Table 1.}




\subsection{Static and dynamic values of PI in ICU patients}

The first study evaluating PI in ICU was published more than 15 years ago by Lima et al. [15]. It evaluated the evolution of PI in healthy volunteers and critically ill patients with poor peripheral perfusion (i.e., capillary refill time $>2 \mathrm{sec}$ or central-to-toe temperature difference $\geq 7^{\circ} \mathrm{C}$ ). In this study, the authors observed a significant exponential relationship between $\mathrm{PI}$ and core-to-toe temperature difference, and significant linear correlation between changes in $\mathrm{PI}$ and in core-to-toe temperature difference. Then, they compared haemodynamic variables between two periods; T1 during poor peripheral perfusion and T2 during normal peripheral perfusion in the 37 critically ill patients under vasoactive medication. They observed a concordant change between T1 and T2 in PI and core-to-toe temperature difference but not with cardiac output, mean arterial pressure or dose of vasoactive agents. In this study, a PI of 1.4 best discriminated between normal and abnormal core-to-toe temperature differences in the critically ill patients (area under the receiver operating characteristics curve $0.91 ; 95 \% \mathrm{Cl} 0.84-0.98, p<0.001)$. In this study, the authors neither looked at the relationship between $\mathrm{PI}$ and capillary refill time, nor between $\mathrm{PI}$ and prognosis (e.g., mortality). This observation suggests that PI could reflect local microcirculation status.

\section{2 $\mathrm{PI}$ and prognosis in ICU and emergency department}

Several studies have shown the negative impact of persisting macrohaemodynamic and/or microcirculatory alterations in patients with acute circulatory failure [48-50]. The hypothesis that severe and/or persistent circulatory alterations in these patients, with higher sympathetic activation, would be reflected by PI values or variations is therefore highly expected. Thus, several studies have confirmed this hypothesis and the prognostic value of 
PI has been evaluated in critically ill patients in different clinical settings (i.e., septic patients, after cardiac arrest, in patients with hypoperfusion, in emergency department and in neonates).

In ICU, in different populations (e.g., septic patients, mechanically ventilated patients...), PI values allow a discrimination between septic patients and non-septic patients and between survivors and non-survivors [51]. Furthermore, PI was strongly associated with ICU mortality $[51,52]$. In patients with hypoperfusion, after 8 hours of resuscitation, PI allowed a better 30days mortality prediction than central venous oxygen saturation $\left(\mathrm{ScvO}_{2}\right)$, lactate and arteriovenous carbon dioxide gradient $\left(\mathrm{P}(\mathrm{v}-\mathrm{a}) \mathrm{CO}_{2}\right)$ [53]. Subgroup analysis suggested that $\mathrm{PI}$ (with a $0.6 \%$ cut-off) could enable to detect patients with the worst prognosis despite $\mathrm{ScvO}_{2}$ normalisation selecting them for adjunctive therapies, notably targeting the microcirculation. This was confirmed in a recent study, in which PI was significantly higher in survivors compared to non-survivors after 6 hours of resuscitation, suggesting that the increase of PI value in the first hours of resuscitation is associated with a better outcome [54]. However, it is impossible to assess whether the difference observed in PI between survivors and non-survivors after initial resuscitation is secondary to an improvement of macrohaemodynamic (i.e., cardiac index) or microcirculation.

The potential prognostic value of peripheral PI after cardiac arrest has been confirmed in a prospective study [55]. In this study, PI was significantly also higher in survivors than in nonsurvivors at day 30 in the first 30 minutes after return to spontaneous circulation. Similar results were again described by the same authors, including a better survival with good neurologic outcome (cerebral performance categories $\leq 2$ ) in patients who had higher PI [56]. However, the authors did not report the cardiac index or other variable such as 
microcirculation variables, making difficult to interpret the PI differences between survivors and non-survivors.

In a prospective study, performances of PI were evaluated for triage of patients admitted to the emergency department for the prediction of mortality at 15 and 30 days [57]. PI was neither predictive of hospital admission, nor associated with mortality in this study. However, this may be explained by a low severity rate and lack of power of PI in this population, compared with ICU patients.

In neonates, a low PI ( $\leq 1.4 \%)$ was found to be accurate for detection of illness severity in the $24 \mathrm{~h}$ after admission [58,59]. More recently, a prospective study gave more data concerning PI values in pre-term infants [60]. Thus, PI seems promising in neonates and infants to easily detect illness severity, and has already made the object of a dedicated review [61].

Although $\mathrm{PI}$ appears efficient to discriminate survivors from non-survivors and septic from non-septic patients in ICU, most studies are retrospective, with PI measurement at different time points and different cut-off values, therefore the clinical use of PI as a prognostic tool remains to be explored.

\subsection{Dynamic changes of PI induced by heat}

More recently, PI was used with a heating challenge to monitor microvascular reactivity in patients presenting a shock [62]. PI at earlobe was significantly higher in healthy volunteers compared to shocked patients. After heating challenge, all the non-septic patients (healthy volunteers, ICU-control patients and non-septic shocked patients, i.e., cardiogenic and haemorrhagic) had similar increase in PI value. PI increase was smaller in septic shock patients after heating challenge than in non-septic patients. In a small population, there was 
a non-significant trend in the evolution of $\mathrm{PI}$ and $\mathrm{PImax} / \mathrm{min}$ after heating challenge in survivors compared to non-survivors. PI variations could enable physicians to discriminate septic from non-septic patients and identify patients with a poor prognosis.

In a prospective observational study including patients under therapeutic hypothermia after cardiac arrest, Van Genderen et al. studied peripheral tissue perfusion, notably using PI before and after rewarming [63]. At admission, PI was not significantly different between survivors and non-survivors. PI became significantly different between survivors and nonsurvivors after rewarming similarly to other microcirculation variable (i.e., perfused capillary density and proportion-perfused vessels) assessed using a side stream dark-field imager. Interestingly, cardiac index was not different between survivors and non-survivors after rewarming, suggesting that PI differences observed between survivors and non-survivors were rather secondary to microcirculation disorders than macrohaemodynamic disorders.

\section{5) Fluid Responsiveness}

The characteristics and main results of discussed studies are detailed in Supplementary Table 1.

\subsection{PI and prediction of fluid responsiveness or fluid removal in ICU}

Beurton et al. assessed whether PI variation during a passive leg-raising (PLR) test could accurately predict fluid responsiveness in ICU patients, mostly under mechanical ventilation [32]. An increase of PI > 9\% induced by PLR predicted an increase of cardiac index $>10 \%$ induced by PLR with a sensitivity of $91 \%(95 \% \mathrm{Cl} 76-98 \%)$, a specificity of $79 \%(95 \% \mathrm{Cl} 63-$ $90 \%)$ and an area under the receiver operating characteristics curve of $0.89(95 \% \mathrm{Cl} 0.8-$ 
0.95). These results were confirmed in another study, which also showed that PI variations during an end-expiratory occlusion test could help to predict positive PLR [33]. However, the cut-off value of relative PI increase during end-expiratory occlusion test was very low (2.5\%), compared to the PI values displayed today in most monitors with most often only one decimal place, and with respect to spontaneous variations in PI. Other authors, using the same relationship between $\mathrm{PI}$ and SV, studied in mechanically ventilated patients during spontaneous breathing trial (using the ratio: PI before weaning trial/PI during weaning trial), to predict weaning failure [64]. They observed that the lack of increase in PI during spontaneous breathing trial was associated with trial failure. This suggests than when PI increases during spontaneous breathing trial, patients may be on the initial vertical part of the Franck-Starling curve, i.e., fluid responders, and thus at lower risk of weaning-induced pulmonary oedema. Furthermore, $\mathrm{PI}$ and $\mathrm{PI}$ variations have been used to predict hypotension during fluid removal under renal replacement therapy [65]. A PI value $\leq 0.82 \%$ predicted hypotension with an area under the receiver operating characteristics curve of 0.8 \pm 0.11 and $\mathrm{PI}$ variation (i.e., decrease) during fluid removal was also predictive of hypotension. As for neuraxial anaesthesia, a low PI may be a witness of high vascular tone and/or low SV, and thus predictive of hypotension due in part to hypovolaemia induced by fluid removal. However, this study included only 23 patients and should be confirmed in a larger cohort.

\subsection{PI respiratory variations and prediction of fluid responsiveness}

The variability of $\mathrm{PI}$ is low during steady-state conditions, under deep general anaesthesia, because general anaesthesia reduces the oscillatory components of the perfusion signal related to sympathetic, myogenic activity and the component modulated by the 
endothelium [66]. Therefore, in this situation PI variations are mainly due to stroke volume variations. PI respiratory variations, called PVI (Pleth Variability Index), have been widely studied in mechanically ventilated patients for the prediction of fluid responsiveness, notably in the operating theatre. The physiological concept is based on tracking PI variations as a surrogate of SV variations (or pulse pressure) induced by positive pressure ventilation. Then the respiratory variations of PI could have nearly the same accuracy to predict fluid responsiveness than pulse pressure variation $[67,68]$.

A recent meta-analysis by Liu et al. highlighted the wide range of PVI cut-off value (from 7\% to $20 \%$ ), depending on the studied population and ventilation settings (e.g., ICU, or, tidal volume, positive end expiratory pressure...) to predict fluid responsiveness [69]. Furthermore, PVI is also (as it is derived from $\mathrm{PI}$ ) largely influenced by the measurement site and seems to be more accurate to predict fluid responsiveness when measured in the forehead, less influenced by vasomotor tone than finger and earlobe [70]. Although a previous study showed reduced volume of intraoperative fluid infused and lactate levels using a PVI-guided fluid management, a recent randomised controlled trial using a haemodynamic peroperative management based on PVI value compared to a standard management did not shorten the duration of hospitalisation [71,72]. Therefore, further studies are needed to assess whether or not PVI value is useful in haemodynamic management in the OT.

PVI could also be an accurate tool to predict fluid responsiveness in children under mechanical ventilation in the operating theatre [73]. Nevertheless, results are conflicting among the studies performed in children and further studies are required to confirm the accuracy of PVI to predict fluid responsiveness in the paediatric population [73-75]. 


\section{6) Perspectives}

After a wide use of PPG for decades for oximetry only, we are experiencing renewed interest and increasing use of PI in anaesthesia, perioperative and critical care. However, some important issues remain to be answered (Figure 3). The characteristics and main results of discussed studies are detailed in Supplementary Table 1.

\subsection{Technical aspects}

Firstly, technical aspects need to be assessed, as the value of PI between manufacturers has never been evaluated and could vary significantly. Algorithms and filters used by manufacturers to process the signal could be sources of distortion of PPG signal, and influence PI performances in various clinical settings. Furthermore, these differences between monitors could also have an impact on the absolute value of $\mathrm{PI}$, and could lead to misinterpretations of this value. Therefore, the adequate cut-off value could be different between manufacturers. Studies assessing PI should always indicate which PPG device has been used.

\subsection{Impact of PI use in haemodynamic management during anaesthesia}

If data show that PI is useful for haemodynamic monitoring, it remains to be explored whether the use of $\mathrm{PI}$ for haemodynamic monitoring would improve the prognosis of patients undergoing surgery. To date, no well-designed studies have been conducted. An interesting study reported the use of an algorithm integrating $\mathrm{PI}$ and pulse pressure variation to guide fluid administration and the use of vasopressors (i.e., in the case of a hypotension event, fluids should be administered if the PI value remained steady during the 15 minutes 
before the hypotension, on the contrary the increase of the PI value should lead to the use of vasopressors). The use of this algorithm was associated with lower duration of arterial hypotension ( $7.7 \pm 5.0 \mathrm{~min}$ vs. $17.1 \pm 10.6 \mathrm{~min}, p=0.003)$ and lower intraoperative fluid administration $(4.3 \pm 1.3 \mathrm{ml} / \mathrm{kg} / \mathrm{h}$ vs. $7.2 \pm 3.3 \mathrm{ml} / \mathrm{kg} / \mathrm{h}, p=0.003)$ [76]. However, this study suffers from several limitations. Among others, there was no algorithm in the control group and the use of an algorithm itself may have favoured an early use of vasopressors rather than fluid challenge. We could assume that another algorithm using other variables without PI may have similar effects.

$\mathrm{PI}$ value before neuraxial anaesthesia predicts hypotension $[44,45]$. Similarly, variations of PI allow real-time detection of arterial hypotension during the induction of general anaesthesia [25]. In these specific situations (i.e., neuraxial anaesthesia, peripheral nerve blockade, induction of anaesthesia, etc.), modifications of $\mathrm{PI}$ values are due predominantly to modifications of the vascular tone. Therefore, in such cases, PI variations are a reliable reflection of sympathetic tone fluctuations. Nevertheless, the impact of PI use in these fields remains to be explored. Indeed, algorithms using PI for early use of vasopressor should be evaluated to prevent hypotension following neuraxial anaesthesia or induction of general anaesthesia.

\subsection{Impact of PI use in haemodynamic management in ICU}

In ICU, PI and its early variations are associated with prognosis. Haemodynamic situations can be complex especially in septic patients, and PI difficult to interpret [35]. In septic patients, PI can also reflect microcirculation disorders as suggested by the lower increase of $\mathrm{PI}$ after heating challenge [62]. However, in patients with preserved microcirculation in ICU, rapid PI variations analyses remain useful in situations in which, among PI determinants, one 
varies predominantly in regard to the others (e.g., PLR, fluid challenge, etc.). Comparing PI value in different measurement sites would possibly provide useful indications about local perfusion; indeed, some territories could remain poorly perfused despite normalisation of macrohaemodynamic status (i.e., MAP, cardiac index, $\mathrm{ScvO}_{2}$ ). We recently described urethral PI monitoring through a modified urinary catheter [77]. Urethral PI monitoring featured a stable, reliable signal capable of measuring urethral tissue perfusion. However, in this study we neither compared urethral PI value with other measurement sites, nor specifically evaluated the effects of therapeutics (i.e., fluid challenge, vasopressors, and blood transfusion) on $\mathrm{PI}$ value. In a proof of concept study, comparing an early peripheral perfusion-guided fluid therapy (using capillary refill time, PI, skin temperature difference between forearm and fingertip and tissue oxygen saturation) vs. standard of care in patients with septic shock, Van Genderen et al. observed that perfusion-guided fluid therapy lead to a trend toward less fluid administration and less organ dysfunction at $72 \mathrm{~h}$ compared with a conventional regimen [78]. Recently, Hernandez et al., in a Bayesian reanalysis of the ANDROMEDA-SHOCK Trial have confirmed this results, concluding that peripheral perfusiontargeted resuscitation (i.e., guided by capillary refill time) may result in lower 28-day mortality when compared with a lactate-targeted resuscitation strategy [79]. Resuscitation algorithms integrating PI for tissue perfusion assessment have also been proposed in ICU [80]. The impact on critically ill patients' outcomes of such algorithms including $\mathrm{PI}$, in association with other resuscitation targets (i.e., cardiac index, mean arterial pressure, $\mathrm{ScvO}_{2}$, capillary refill time) should be tested in large randomised controlled trials. However, in these complex situations (e.g., septic shock, haemorrhagic shock...), several determinants of PI may vary simultaneously and abruptly (i.e., increase in vascular tone due to vasopressors, increase in stoke volume due to fluid loading and/or inotropic drug 
introduction). This may contribute to abrupt and complex changes in PI. In these situations, PI variations are more difficult to interpret, and should probably be used as an early signal that should prompt reassessment of haemodynamic status, including stroke volume to accurately interpret PI variations.

\section{7) Conclusion}

$\mathrm{PI}$ is a PPG-derived variable, already given by most devices, measuring perfusion at crossroads between central and peripheral perfusion. It appears to be a useful, non-invasive additional tool for haemodynamic monitoring in anaesthesiology, perioperative and critical care for clinicians. Knowing its determinants is crucial to be able to interpret its variations. Whether the use of $\mathrm{PI}$ in resuscitation algorithms would improve patient outcomes remains to be explored. 


\section{References:}

[1] Aoyagi T. Pulse oximetry: its invention, theory, and future. Journal of Anesthesia 2003;17:25966. https://doi.org/10.1007/s00540-003-0192-6.

[2] Alian AA, Shelley KH. Photoplethysmography. Best Practice \& Research Clinical Anaesthesiology 2014;28:395-406. https://doi.org/10.1016/j.bpa.2014.08.006.

[3] Reisner A, Shaltis PA, McCombie D, Asada HH. Utility of the photoplethysmogram in circulatory monitoring. Anesthesiology 2008;108:950-8. https://doi.org/10.1097/ALN.0b013e31816c89e1.

[4] Elgendi M. Optimal Signal Quality Index for Photoplethysmogram Signals. Bioengineering 2016;3:21. https://doi.org/10.3390/bioengineering3040021.

[5] Korhonen I, Yli-Hankala A. Photoplethysmography and nociception. Acta Anaesthesiologica Scandinavica 2009;53:975-85. https://doi.org/10.1111/j.1399-6576.2009.02026.x.

[6] Allen J. Photoplethysmography and its application in clinical physiological measurement. Physiological Measurement 2007;28:R1-39. https://doi.org/10.1088/0967-3334/28/3/R01.

[7] Murray WB, Foster PA. The peripheral pulse wave: information overlooked. J Clin Monit 1996;12:365-77.

[8] Plethsmographic waveform shapes displayed by oximeters-Technical note 2008.

[9] PHILIPS. IntelliVue Patient Monitor Philips FAST-SpO2: Application Note n.d.

[10] Nijboer JA, Dorlas JC, Mahieu HF. Photoelectric plethysmography--some fundamental aspects of the reflection and transmission method. Clin Phys Physiol Meas 1981;2:205-15.

[11] Fine J, Branan KL, Rodriguez AJ, Boonya-ananta T, Ajmal, Ramella-Roman JC, et al. Sources of Inaccuracy in Photoplethysmography for Continuous Cardiovascular Monitoring. Biosensors 2021;11:126. https://doi.org/10.3390/bios11040126.

[12] Goldman JM, Petterson MT, Kopotic RJ, Barker SJ. Masimo signal extraction pulse oximetry. Journal of Clinical Monitoring and Computing 2000;16:475-483.

[13] Sang-Soo Oak, Praveen Aroul. How to Design Peripheral Oxygen Saturation (SpO2) and Optical Heart Rate Monitoring (OHRM) Systems Using the AFE4403. 2015.

[14] Colquhoun DA, Forkin KT, Durieux ME, Thiele RH. Ability of the Masimo pulse CO-Oximeter to detect changes in hemoglobin. J Clin Monit Comput 2012;26:69-73. https://doi.org/10.1007/s10877-012-9335-3.

[15] Lima AP, Beelen P, Bakker J. Use of a peripheral perfusion index derived from the pulse oximetry signal as a noninvasive indicator of perfusion. Critical Care Medicine 2002;30:12101213.

[16] Keller G, Cassar E, Desebbe O, Lehot J-J, Cannesson M. Ability of pleth variability index to detect hemodynamic changes induced by passive leg raising in spontaneously breathing volunteers. Crit Care 2008;12:R37. https://doi.org/10.1186/cc6822.

[17] Nitzan M, Faib I, Friedman H. Respiration-induced changes in tissue blood volume distal to occluded artery, measured by photoplethysmography. J Biomed Opt 2006;11:040506. https://doi.org/10.1117/1.2236285.

[18] Shelley KH. Photoplethysmography: beyond the calculation of arterial oxygen saturation and heart rate. Anesth Analg 2007;105:S31-36, tables of contents. https://doi.org/10.1213/01.ane.0000269512.82836.c9.

[19] Jeong I, Jun S, Um D, Oh J, Yoon H. Non-Invasive Estimation of Systolic Blood Pressure and Diastolic Blood Pressure Using Photoplethysmograph Components. Yonsei Med J 2010;51:345. https://doi.org/10.3349/ymj.2010.51.3.345.

[20] Galvin EM, Niehof S, Verbrugge SJ, Maissan I, Jahn A, Klein J, et al. Peripheral Flow Index Is a Reliable and Early Indicator of Regional Block Success: Anesthesia \& Analgesia 2006;103:23943. https://doi.org/10.1213/01.ane.0000220947.02689.9f.

[21] Ginosar Y, Weiniger CF, Meroz Y, Kurz V, Bdolah-Abram T, Babchenko A, et al. Pulse oximeter perfusion index as an early indicator of sympathectomy after epidural anesthesia. Acta 
Anaesthesiologica Scandinavica 2009;53:1018-26. https://doi.org/10.1111/j.1399-

6576.2009.01968.x.

[22] Kus A, Gurkan Y, Gormus SK, Solak M, Toker K. Usefulness of perfusion index to detect the effect of brachial plexus block. Journal of Clinical Monitoring and Computing 2013;27:325-8. https://doi.org/10.1007/s10877-013-9439-4.

[23] Uemura A, Yagihara M, Miyabe M. Pulse oxymeter perfusion index as a predictor for the effect of pediatric epidural block. Anesthesiology 2006;105:A1354.

[24] Sebastiani A, Philippi L, Boehme S, Closhen D, Schmidtmann I, Scherhag A, et al. Perfusion index and plethysmographic variability index in patients with interscalene nerve catheters. Canadian Journal of Anesthesia/Journal Canadien d'anesthésie 2012;59:1095-101. https://doi.org/10.1007/s12630-012-9796-3.

[25] Coutrot M, Joachim J, Dépret F, Millasseau S, Nougué H, Matéo J, et al. Noninvasive continuous detection of arterial hypotension during induction of anaesthesia using a photoplethysmographic signal: proof of concept. British Journal of Anaesthesia 2019;122:60512. https://doi.org/10.1016/j.bja.2019.01.037.

[26] Corsini I, Cecchi A, Coviello C, Dani C. Perfusion index and left ventricular output correlation in healthy term infants. Eur J Pediatr 2017;176:1013-8. https://doi.org/10.1007/s00431-0172920-1.

[27] Janaillac M, Beausoleil TP, Barrington KJ, Raboisson M-J, Karam O, Dehaes M, et al. Correlations between near-infrared spectroscopy, perfusion index, and cardiac outputs in extremely preterm infants in the first $72 \mathrm{~h}$ of life. Eur J Pediatr 2018;177:541-50. https://doi.org/10.1007/s00431-018-3096-z.

[28] Takahashi S, Kakiuchi S, Nanba Y, Tsukamoto K, Nakamura T, Ito Y. The perfusion index derived from a pulse oximeter for predicting low superior vena cava flow in very low birth weight infants. Journal of Perinatology 2010;30:265-269.

[29] van Genderen ME, Bartels SA, Lima A, Bezemer R, Ince C, Bakker J, et al. Peripheral Perfusion Index as an Early Predictor for Central Hypovolaemia in Awake Healthy Volunteers: Anesthesia \& Analgesia 2013;116:351-6. https://doi.org/10.1213/ANE.0b013e318274e151.

[30] Tapar H, Karaman S, Dogru S, Karaman T, Sahin A, Tapar GG, et al. The effect of patient positions on perfusion index. BMC Anesthesiol 2018;18:111. https://doi.org/10.1186/s12871018-0571-z.

[31] Højlund J, Agerskov M, Clemmesen CG, Hvolris LE, Foss NB. The Peripheral Perfusion Index tracks systemic haemodynamics during general anaesthesia. J Clin Monit Comput 2019. https://doi.org/10.1007/s10877-019-00420-x.

[32] Beurton A, Teboul J-L, Gavelli F, Gonzalez FA, Girotto V, Galarza L, et al. The effects of passive leg raising may be detected by the plethysmographic oxygen saturation signal in critically ill patients. Crit Care 2019;23:19. https://doi.org/10.1186/s13054-019-2306-z.

[33] Beurton A, Gavelli F, Teboul J-L, De Vita N, Monnet X. Changes in the Plethysmographic Perfusion Index During an End-Expiratory Occlusion Detect a Positive Passive Leg Raising Test*. Critical Care Medicine 2021;49:e151-60. https://doi.org/10.1097/CCM.0000000000004768.

[34] Lian $\mathrm{H}$, Wang $X$, Zhang $\mathrm{Q}$, Zhang $\mathrm{H}$, Liu $\mathrm{D}$. Changes in perfusion can detect changes in the cardiac index in patients with septic shock. J Int Med Res 2020;48:030006052093167. https://doi.org/10.1177/0300060520931675.

[35] He H-W, Liu W-L, Zhou X, Long Y, Liu D-W. Effect of mean arterial pressure change by norepinephrine on peripheral perfusion index in septic shock patients after early resuscitation. Chin Med J (Engl) 2020;133:2146-52. https://doi.org/10.1097/CM9.0000000000001017.

[36] Charkoudian N. Mechanisms and modifiers of reflex induced cutaneous vasodilation and vasoconstriction in humans. Journal of Applied Physiology 2010;109:1221-8. https://doi.org/10.1152/japplphysiol.00298.2010.

[37] Hodges GJ, Johnson JM. Adrenergic control of the human cutaneous circulation. Appl Physiol Nutr Metab 2009;34:829-39. https://doi.org/10.1139/H09-076. 
[38] Awad AA, Ghobashy MA, Ouda W, Stout RG, Silverman DG, Shelley KH. Different responses of ear and finger pulse oximeter wave form to cold pressor test. Anesth Analg 2001;92:1483-6. https://doi.org/10.1097/00000539-200106000-00026.

[39] Shelley KH, Tamai D, Jablonka D, Gesquiere M, Stout RG, Silverman DG. The Effect of Venous Pulsation on the Forehead Pulse Oximeter Wave Form as a Possible Source of Error in Spo2 Calculation: Anesthesia \& Analgesia 2005;100:743-7. https://doi.org/10.1213/01.ANE.0000145063.01043.4B.

[40] Kakazu CZ, Chen B-J, Kwan W-F. Masimo set technology using perfusion index is a sensitive indicator for epidural onset. Anesthesiology 2005;103:A576.

[41] Xu Z, Zhang J, Xia Y, Deng X. Accuracy of pulse oximeter perfusion index in thoracic epidural anesthesia under basal general anesthesia. International Journal of Clinical and Experimental Medicine 2014;7:1728.

[42] Abdelhamid B, Emam M, Mostafa M, Hasanin A, Awada W, Rady A, et al. The ability of perfusion index to detect segmental ulnar nerve sparing after supraclavicular nerve block. J Clin Monit Comput 2019. https://doi.org/10.1007/s10877-019-00443-4.

[43] Kim D, Jeong JS, Park MJ, Ko JS. The effect of epinephrine on the perfusion index during ultrasound-guided supraclavicular brachial plexus block: a randomized controlled trial. Sci Rep 2020;10:11585. https://doi.org/10.1038/s41598-020-68475-4.

[44] Toyama S, Kakumoto M, Morioka M, Matsuoka K, Omatsu H, Tagaito Y, et al. Perfusion index derived from a pulse oximeter can predict the incidence of hypotension during spinal anaesthesia for Caesarean delivery. British Journal of Anaesthesia 2013;111:235-41. https://doi.org/10.1093/bja/aet058.

[45] Duggappa D, Lokesh M, Dixit A, Paul R, Raghavendra Rao R, Prabha P. Perfusion index as a predictor of hypotension following spinal anaesthesia in lower segment caesarean section. Indian J Anaesth 2017;61:649. https://doi.org/10.4103/ija.IJA_429_16.

[46] Hager H., Church S., Mandadi G., Pulley D. The Perfusion Index as Measured by a Pulse Oximeter Indicates Pain Stimuli in Anesthetized Volunteers. Anesthesiology 2004;101:A514.

[47] Agerskov M, Thusholdt ANW, Holm-Sørensen H, Wiberg S, Meyhoff CS, Højlund J, et al. Association of the intraoperative peripheral perfusion index with postoperative morbidity and mortality in acute surgical patients: a retrospective observational multicentre cohort study. British Journal of Anaesthesia 2021:S0007091221003561. https://doi.org/10.1016/j.bja.2021.06.004.

[48] Sakr Y, Dubois M-J, De Backer D, Creteur J, Vincent J-L. Persistent microcirculatory alterations are associated with organ failure and death in patients with septic shock. Crit Care Med 2004;32:1825-31.

[49] Top APC, Ince C, de Meij N, van Dijk M, Tibboel D. Persistent low microcirculatory vessel density in nonsurvivors of sepsis in pediatric intensive care*: Critical Care Medicine 2011;39:8-13. https://doi.org/10.1097/CCM.0b013e3181fb7994.

[50] Ait-Oufella H, Lemoinne S, Boelle PY, Galbois A, Baudel JL, Lemant J, et al. Mottling score predicts survival in septic shock. Intensive Care Medicine 2011;37:801-7. https://doi.org/10.1007/s00134-011-2163-y.

[51] $\mathrm{He} H$, Liu $D$, Long $Y$, Wang $X$. The peripheral perfusion index and transcutaneous oxygen challenge test are predictive of mortality in septic patients after resuscitation. Crit Care 2013;17:R116. https://doi.org/10.1186/cc12788.

[52] Su L, Zhang R, Zhang Q, Xu Q, Zhou X, Cui N, et al. The Effect of Mechanical Ventilation on Peripheral Perfusion Index and Its Association With the Prognosis of Critically III Patients: Critical Care Medicine 2019;47:685-90. https://doi.org/10.1097/CCM.0000000000003661.

[53] He H, Long $Y$, Liu D, Wang X, Zhou X. Clinical classification of tissue perfusion based on the central venous oxygen saturation and the peripheral perfusion index. Crit Care 2015;19:330. https://doi.org/10.1186/s13054-015-1057-8. 
[54] Mongkolpun W, Orbegozo D, Cordeiro CPR, Franco CJCS, Vincent J-L, Creteur J. Alterations in Skin Blood Flow at the Fingertip Are Related to Mortality in Patients With Circulatory Shock: Critical Care Medicine 2020;48:443-50. https://doi.org/10.1097/CCM.0000000000004177.

[55] Savastano S, Baldi E, Molinari S, Canevari F, Corazza V, Cacciatore E, et al. Post ROSC pulseoximetry derived perfusion index and thirty days survival after out-of-hospital cardiac arrest. New insights from the Pavia CARe (Pavia Cardiac Arrest Registry). Resuscitation 2017;117:e1920. https://doi.org/10.1016/j.resuscitation.2017.05.027.

[56] Savastano S, Baldi E, Contri E, De Pirro A, Sciutti F, Compagnoni S, et al. Post-ROSC peripheral perfusion index discriminates 30-day survival after out-of-hospital cardiac arrest. Intern Emerg Med 2020. https://doi.org/10.1007/s11739-020-02430-z.

[57] Oskay A, Eray O, Dinç SE, Aydın AG, Eken C. Prognosis of Critically III Patients in the ED and Value of Perfusion Index Measurement: A Cross-Sectional Study. The American Journal of Emergency Medicine 2015;33:1042-4. https://doi.org/10.1016/j.ajem.2015.04.033.

[58] De Felice C, Latini G, Vacca P, Kopotic RJ. The pulse oximeter perfusion index as a predictor for high illness severity in neonates. European Journal of Pediatrics 2002;161:561-2. https://doi.org/10.1007/s00431-002-1042-5.

[59] Richardson DK, Gray JE, McCormick MC, Workman K, Goldmann DA. Score for Neonatal Acute Physiology: a physiologic severity index for neonatal intensive care. Pediatrics 1993;91:617-23.

[60] Alderliesten T, Lemmers PMA, Baerts W, Groenendaal F, van Bel F. Perfusion Index in Preterm Infants during the First 3 Days of Life: Reference Values and Relation with Clinical Variables. Neonatology 2015;107:258-65. https://doi.org/10.1159/000370192.

[61] Piasek CZ, Van Bel F, Sola A. Perfusion index in newborn infants: a noninvasive tool for neonatal monitoring. Acta Paediatr 2014;103:468-73. https://doi.org/10.1111/apa.12574.

[62] Vallée F, Nougué H, Mari A, Vodovar N, Dubreuil G, Damoisel C, et al. Variations of Cutaneous Capnometry and Perfusion Index During a Heating Challenge is Early Impaired in Septic Shock and Related to Prognostic in Non-Septic Shock: SHOCK 2019;51:585-92. https://doi.org/10.1097/SHK.0000000000001216.

[63] van Genderen ME, Lima A, Akkerhuis M, Bakker J, van Bommel J. Persistent peripheral and microcirculatory perfusion alterations after out-of-hospital cardiac arrest are associated with poor survival*: Critical Care Medicine 2012;40:2287-94. https://doi.org/10.1097/CCM.0b013e31825333b2.

[64] Lotfy A, Hasanin A, Rashad M, Mostafa M, Saad D, Mahmoud M, et al. Peripheral perfusion index as a predictor of failed weaning from mechanical ventilation. J Clin Monit Comput 2020. https://doi.org/10.1007/s10877-020-00483-1.

[65] Klijn E, Groeneveld ABJ, van Genderen ME, Betjes M, Bakker J, van Bommel J. Peripheral Perfusion Index Predicts Hypotension during Fluid Withdrawal by Continuous Veno-Venous Hemofiltration in Critically III Patients. Blood Purif 2015;40:92-8. https://doi.org/10.1159/000381939.

[66] Landsverk SA, Kvandal P, Bernjak A, Stefanovska A, Kirkeboen KA. The Effects of General Anesthesia on Human Skin Microcirculation Evaluated by Wavelet Transform: Anesthesia \& Analgesia 2007;105:1012-9. https://doi.org/10.1213/01.ane.0000281932.09660.96.

[67] Chu H, Wang $Y$, Sun $Y$, Wang G. Accuracy of pleth variability index to predict fluid responsiveness in mechanically ventilated patients: a systematic review and meta-analysis. J Clin Monit Comput 2016;30:265-74. https://doi.org/10.1007/s10877-015-9742-3.

[68] Yang X, Du B. Does pulse pressure variation predict fluid responsiveness in critically ill patients? A systematic review and meta-analysis. Crit Care 2014;18:650. https://doi.org/10.1186/s13054014-0650-6.

[69] Liu T, Xu C, Wang M, Niu Z, Qi D. Reliability of pleth variability index in predicting preload responsiveness of mechanically ventilated patients under various conditions: a systematic review and meta-analysis. BMC Anesthesiol 2019;19:67. https://doi.org/10.1186/s12871-0190744-4. 
[70] Desgranges F-P, Desebbe O, Ghazouani A, Gilbert K, Keller G, Chiari P, et al. Influence of the site of measurement on the ability of plethysmographic variability index to predict fluid responsiveness. British Journal of Anaesthesia 2011;107:329-35. https://doi.org/10.1093/bja/aer165.

[71] Fischer M-O, Lemoine S, Tavernier B, Bouchakour C-E, Colas V, Houard M, et al. Individualized Fluid Management Using the Pleth Variability Index: A Randomized Clinical Trial. Anesthesiology 2020;133:31-40. https://doi.org/10.1097/ALN.0000000000003260.

[72] Forget P, Lois F, de Kock M. Goal-Directed Fluid Management Based on the Pulse OximeterDerived Pleth Variability Index Reduces Lactate Levels and Improves Fluid Management: Anesthesia \& Analgesia 2010:1. https://doi.org/10.1213/ANE.0b013e3181eb624f.

[73] Desgranges F-P, Evain J-N, Pereira de Souza Neto E, Raphael D, Desebbe O, Chassard D. Does the plethysmographic variability index predict fluid responsiveness in mechanically ventilated children? A meta-analysis. British Journal of Anaesthesia 2016;117:409-10. https://doi.org/10.1093/bja/aew245.

[74] Pereira de Souza Neto E, Grousson S, Duflo F, Ducreux C, Joly H, Convert J, et al. Predicting fluid responsiveness in mechanically ventilated children under general anaesthesia using dynamic parameters and transthoracic echocardiography. British Journal of Anaesthesia 2011;106:85664. https://doi.org/10.1093/bja/aer090.

[75] Chen P, Chan K, Liao M, Wu C. Accuracy of dynamic preload variables for predicting fluid responsiveness in patients with pediatric liver cirrhosis: A prospective study. Pediatr Anaesth 2020;30:455-61. https://doi.org/10.1111/pan.13819.

[76] Godai K, Matsunaga A, Kanmura Y. The effects of hemodynamic management using the trend of the perfusion index and pulse pressure variation on tissue perfusion: a randomized pilot study. JA Clin Rep 2019;5:72. https://doi.org/10.1186/s40981-019-0291-5.

[77] Dépret F, Leone M, Duclos G, Futier E, Montagne M, Legrand M, et al. Monitoring tissue perfusion: a pilot clinical feasibility and safety study of a urethral photoplethysmographyderived perfusion device in high-risk patients. J Clin Monit Comput 2019. https://doi.org/10.1007/s10877-019-00414-9.

[78] van Genderen ME, Engels N, van der Valk RJP, Lima A, Klijn E, Bakker J, et al. Early Peripheral Perfusion-guided Fluid Therapy in Patients with Septic Shock. Am J Respir Crit Care Med 2015;191:477-80. https://doi.org/10.1164/rccm.201408-1575LE.

[79] Zampieri FG, Damiani LP, Bakker J, Ospina-Tascón GA, Castro R, Cavalcanti AB, et al. Effects of a Resuscitation Strategy Targeting Peripheral Perfusion Status versus Serum Lactate Levels among Patients with Septic Shock. A Bayesian Reanalysis of the ANDROMEDA-SHOCK Trial. Am J Respir Crit Care Med 2020;201:423-9. https://doi.org/10.1164/rccm.201905-09680C.

[80] Hariri G, Joffre J, Leblanc G, Bonsey M, Lavillegrand J-R, Urbina T, et al. Narrative review: clinical assessment of peripheral tissue perfusion in septic shock. Ann Intensive Care 2019;9:37. https://doi.org/10.1186/s13613-019-0511-1. 
Table 1: Determinants of Perfusion Index and artifacts

\section{Physiological and pathophysiological determinants of Perfusion Index}

\section{Systemic factors}

\section{Local factors}

- Obliterant arteriopathy

Volaemia and venous return*

- Diastolic function and inotropism *

- Valvulopathy*

- Vascular tone*(sympathetic, parasympathetic and non-adrenergic/non-cholinergic tones)

- Arterial stiffness*

- Vasoactive and cardiac medications*
- Position of the limb in relation to the heart

- Vascular compression

- Local temperature exposure

- Local arterial compliance

- Local vascular tone

\section{Factors influencing DC}

- Vascular tone* (notably venous tone)

- Volaemia*
- Position of the limb

- Body position

- Vascular compression

- Soft tissues compression

- Local vascular tone

\section{Artifacts that may influence $A C$ and $D C$}

- External light

- Nail polish
- Tissues and vascular extrinsic compressions

- Probe and/or patient movements

Numerous intrinsic or extrinsic factors influence $A C$ and/or DC, and therefore their ratio, i.e. Perfusion Index. The main determinants are stroke volume and vascular tone, themselves influenced by many factors. Determinants of stroke volume $(*)$ all may influence AC and PI. Volaemia, external temperature, stress, nociception, and medications (e.g. norepinephrine, vasodilator effect of anaesthetic drugs, etc.) are determinants of vascular tone and therefore of Perfusion Index.

* Determinants of stroke volume

AC: alternating current; DC: direct current 
Figure 1: Principles of photoplethysmography and PI calculation

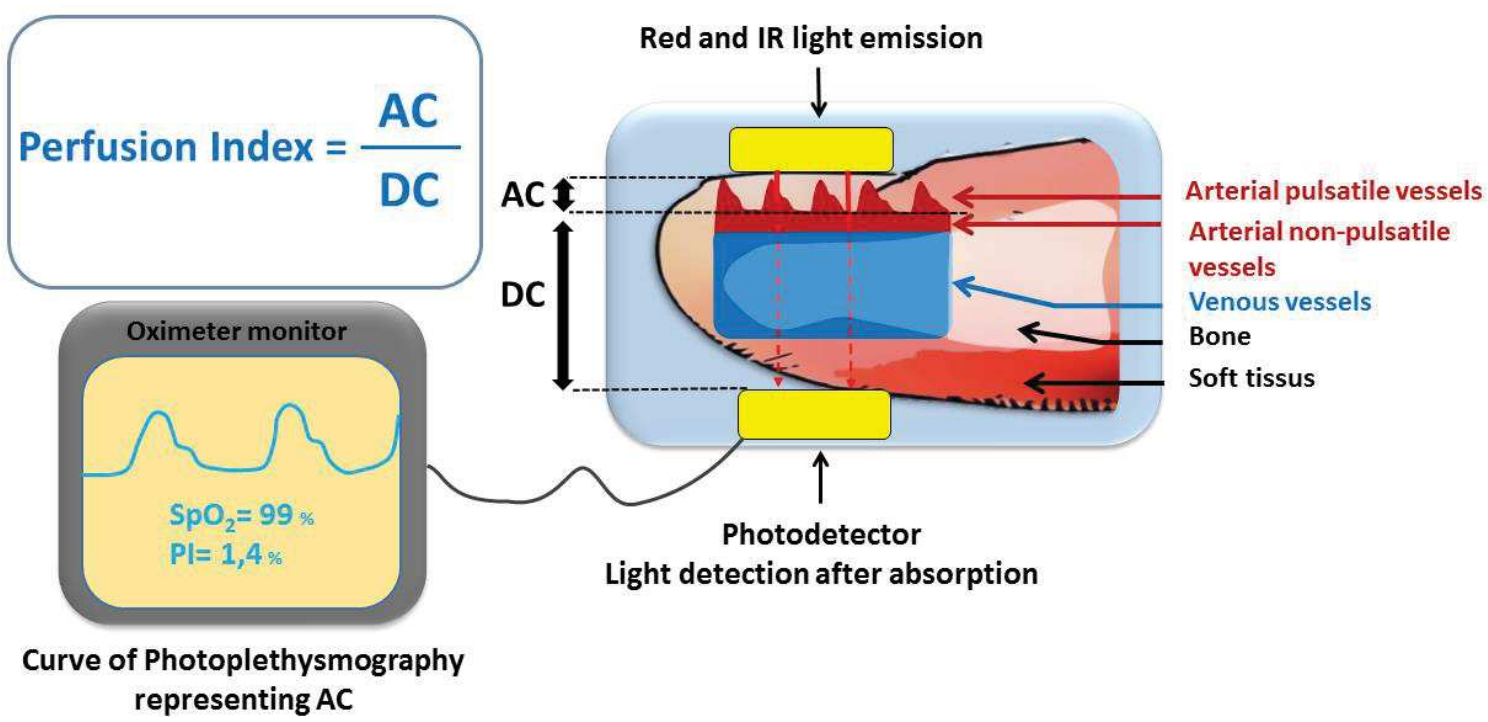

Figure 1 represents the principles of PPG and PI calculation. AC corresponds to the variation of red and IR lights absorption related to the variation of diameters of pulsatile vessels (i.e. arrowed arterial pulsatile vessels on the figure). DC corresponds to the light absorption of: arterial non-pulsatile vessels, venous vessels, bone and soft tissues. PI value is calculated as the $A C / D C$ ratio.

AC: alternating current; DC: direct current; IR: infrared light PI: perfusion index; PPG: photoplethysmography 
Figure 2: Determinants of PI and typical clinical situations

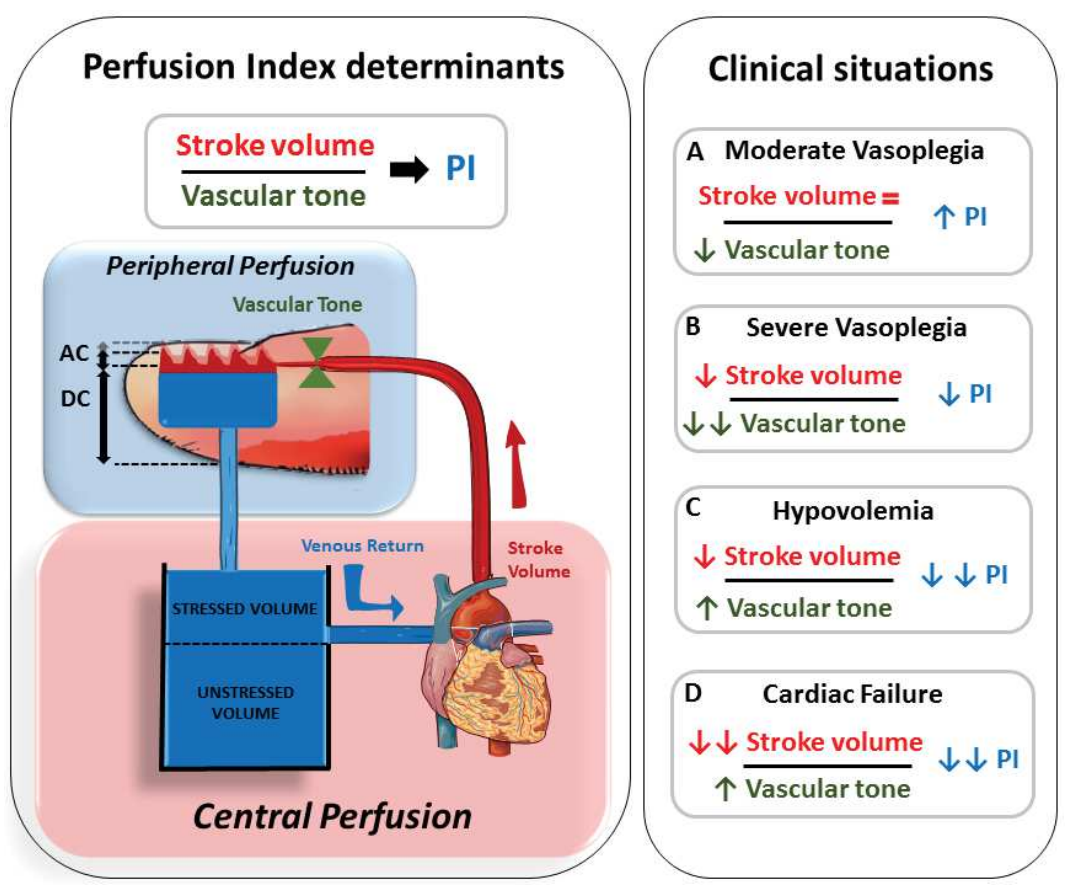

PI results from the local perfusion balance between peripheral determinants, mainly vascular tone and central determinants, i.e. SV. Situation A represents the components of moderate vasoplegia: decreased vascular tone with single arterial vasodilatation, without significant variations in SV, resulting in an increase in PI. If vasoplegia becomes severe (situation B), with arterial and venous vasodilatation, the drop of stressed volume, venous return and thus SV leads to a secondary decrease in $\mathrm{PI}$, despite the decrease of vascular tone.

In situation C and D, i.e. hypovolaemia and cardiogenic shock, the drop of SV and the increase in vascular tone both contributes to a decrease in PI.

AC: alternating current; DC: direct current; PI: perfusion index; SV: stoke volume 
Figure 3: Summary of clinical usefulness and perspectives of PI use in anaesthesia and critical

care

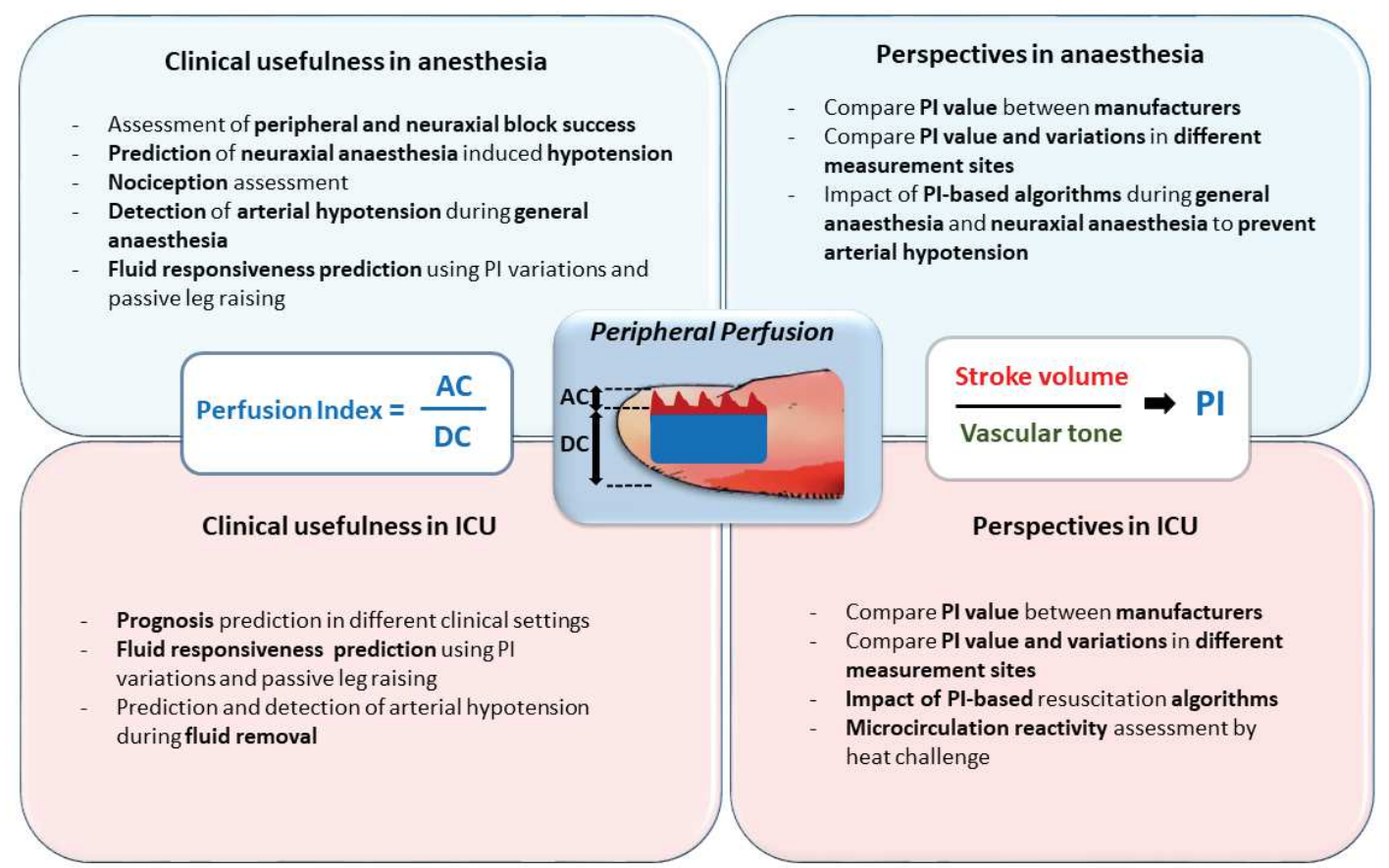

AC: alternating current; DC: direct current; IR: infrared light; PI: perfusion index; PPG: photoplethysmography; AC: alternating current; DC: direct current 